\title{
Editor's Notes and Instructions to Authors
}

CEREAL RESEARCH COMMUNICATIONS (Cereal Res. Comm.) is a quarterly published by the Cereal Research Non-Profit Company, Managing Director: J. FRANK, D.Sc., Address: H-6701 Szeged, P.O.Box 391., Hungary, Phone: 36-62-435235, Fax: 36-62-434163, E-mail: h9865ker@ella.hu

CEREAL RESEARCH COMMUNICATIONS deals with breeding, genetical, agrotechnical, and crop physiological investigations of primarily wheat, maize, barley, oats, sorghum as well as oil crops. Publication is fast. Papers are duplicated in their original typed form whenever possible eliminating printing errors.

Only original papers and short reviews in English can be published as described below. Book reviews and books intended for reviewing are also welcomed if they deal with the above topics.

Camera ready single spaced manuscripts are requested in two copies with a clear, unwrinkled top copy. To facilitate our duplication procedure and save space, we ask that the text be typed in a frame of $16 \times 22 \mathrm{~cm}(6.3 \times 8.2$ inches) on 20-22 $\times 28-30$ cm (A4) white paper. Font size must be at least 12 because text will be reproduced at $75 \%$ of the original size. Figures and table should be compactly arranged and inserted in the text. Coloured figures, photos are accepted for the cost of the authors. Manuscripts should not exceed eight pages in length including figures, tables and references. The articles should be divided into the following sections: Summary followed by a list of index words, Introduction, Materials and Methods, Results, Discussion, References.

Articles may be sent to any member of the Editorial Board, or directly to the Managing Editor. All manuscripts will be reviewed before acceptance. However, even recommended papers may be rejected due to lack of space. Full name and mailing address of the corresponding authors should be given. Fax or E-mail address is also requested, if available.

After acceptance, submission of the final version on disk is encouraged, accompanied by a hard copy printout. For text use Word for Windows 6.0 or earlier versions.

The annual subscription fee of Cereal Research Communications is 100 US \$ per volume (4 issues) both for qualified institutions and individuals. For a subscription please send your inquiries to the Managing Editor.

Comments are welcomed, for we wish to develop our journal into a periodical which is of the greatest possible value to experts in this field. 